\title{
Is consanguinity a relevanit factor in psychosis? The case of Easter Island (RapaNuí).
}

MD. Fontanez $\mathbf{K}^{\mathbf{1 , 3}}$, MD. Vasquez $\mathrm{P}^{2}$

1. Psychiatrist at "Dr. J. Horwitz" Psychiatric Institute, Santiago, Chile. 3. Universidad de Santiago, School of Medicine, Santiago, Chile. 2. Psychiatrist Resident,

Universidad de Santiago, "Dr. J. Horwitz" Psychiatric Institute, Santiago, Chile.

Objectives: Make a review about consanguinity and mental illness, especially psychosis, and relate the information with the reality of Easter Island.

Background: A consanguineous marriage is defined as the union of a couple related as second cousins or closer. They represent $10.4 \%$ of the population. It has been described in isolated populations, like Easter Island. It is still possible to observe families of ancestral lineage, being striking the development of severe psychiatric illness in recent years.

Methodology: We collated studies found via searches on databases (PubMed, MedLine, ScienceDirect, Scielo), using relevant terms ("Rapanui", "Polynesia”, "Psychosis", "Easer Island", "consanguinity", endogamy"). We gather information about islanders who are consultants in the Psychiatric Institute in Santiago, Chile, between 2013-2018.

Results: A few studies confirm an association between consanguinity and mental disorders, with a higher risk of psychosis (Table 1). Endogamy in RapaNui was decreasing. In 2006, an analysis found a low level, suggesting strategy used to avoid marriage between close relatives. We found few epidemiological records and no information on outpatient care. In 2015 , $3.3 \%$ hospitalizations were due to mental illness. There is high frequency of domestic violence and drug abuse. At the Psychiatric Institute, nineteen islanders were received (Table 2). Several native surnames in common were found.

Discussion: It is urgent to study the psychiatric illness in RapaNui and to carry out a study on consanguinity, considering that in some subpopulation it has been demonstrated that more distant genetic relationships may also be important.

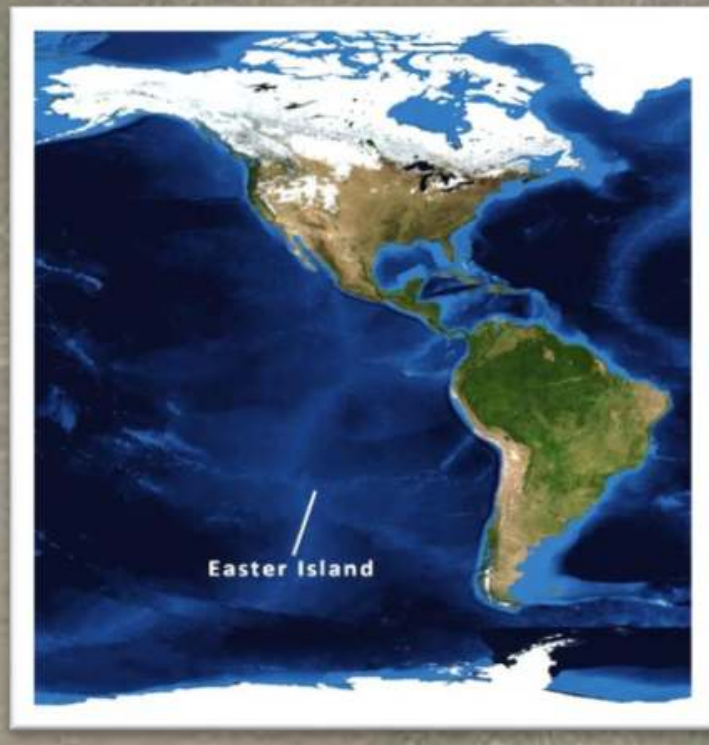

Table 1. Example of studies about inbreading and mental illness.

\begin{tabular}{|c|c|c|}
\hline $\begin{array}{l}\text { Study } \\
\text { design }\end{array}$ & Results & Publication \\
\hline $\begin{array}{l}\text { Cross- } \\
\text { sectional } \\
\text { study }\end{array}$ & $\begin{array}{l}\text { Parental consanguinity was elevated among the } \\
\text { patients with schizophrenia ( } 41.3 \% \text { ) with a } \\
\text { higher mean coefficient of inbreeding than in } \\
\text { non-schizophrenic subjects ( } 28.7 \% \text { ) }\end{array}$ & $\begin{array}{l}\text { Bener } A \\
2012, \\
\text { Abdulbari } B \\
2012\end{array}$ \\
\hline $\begin{array}{l}\text { Case } \\
\text { report }\end{array}$ & Puerperal psychosis associated in three sisters. & $\begin{array}{l}\text { Craddock, } \\
1994\end{array}$ \\
\hline $\begin{array}{l}\text { Case } \\
\text { report }\end{array}$ & $\begin{array}{l}\text { A consanguineus family affected by bipolar } \\
\text { disorder associated with more aggressive } \\
\text { episodes, early onset, and treatment relapse. }\end{array}$ & Aadil M, 2017 \\
\hline $\begin{array}{l}\text { Case - } \\
\text { control }\end{array}$ & $\begin{array}{l}\text { Association with depression in South India, with } \\
\text { odds ratio of } 5.66\end{array}$ & $\begin{array}{l}\text { Sathyanaraya } \\
\text { na Rao, } T \text {. } \\
2009\end{array}$ \\
\hline $\begin{array}{l}\text { Case - } \\
\text { control }\end{array}$ & $\begin{array}{l}\text { In late onset cases of bipolar disorder, this was } \\
\text { lower for offspring of consanguineous than } \\
\text { unrelated marriages. }\end{array}$ & $\begin{array}{l}\text { Saadat } M \\
2012\end{array}$ \\
\hline Review & $\begin{array}{l}\text { Consanguinity associated with mental } \\
\text { retardation in different studies. }\end{array}$ & $\begin{array}{l}\text { Matthew C, } \\
2006\end{array}$ \\
\hline $\begin{array}{l}\text { Case - } \\
\text { control }\end{array}$ & $\begin{array}{l}\text { The estimated mean inbreeding coefficient }(\alpha) \\
\text { for cases of drug abuse }(0.0179) \text { was higher } \\
\text { than that of the control group }(0.0114) \text {. }\end{array}$ & $\begin{array}{l}\text { Mostafa S } \\
2010\end{array}$ \\
\hline
\end{tabular}

Table 2. Description of islanders who consulted to Psychiatric Institute in Santiago, Chile, between 2013-2018.

\begin{tabular}{|c|c|c|c|c|}
\hline Subjet & Surnames & Age (years) & Gender & Diagnosis \\
\hline 1 & $A X$ & 29 & Male & Bipolar disorder \\
\hline 2 & $\mathrm{AA}$ & 24 & Female & Personality disorder \\
\hline 3 & $A B$ & 49 & Female & Depresive episode \\
\hline 4 & $A X$ & 27 & Male & Personality disorder \\
\hline 5 & CD & 35 & Male & Alcohol abuse \\
\hline 6 & $X E$ & 35 & Male & Bipolar disorder \\
\hline 7 & $X E$ & 36 & Male & Schizophrenia \\
\hline 8 & $X F$ & 31 & Male & Bipolar disorder \\
\hline 9 & $\mathrm{GX}$ & 29 & Male & Schizophrenia \\
\hline 10 & $F G$ & 37 & Male & Schizophrenia \\
\hline 11 & $\mathrm{GH}$ & 45 & Female & Personality disorder \\
\hline 12 & $\mathrm{XH}$ & 39 & Female & Personality disorder \\
\hline 13 & $\mathrm{HB}$ & 22 & Male & Schizophrenia \\
\hline 14 & $x J$ & 30 & Male & Schizophrenia \\
\hline 15 & $\mathrm{KX}$ & 27 & Male & Bipolar disorder \\
\hline 16 & LX & 26 & Female & Personality disorder \\
\hline 17 & $F G$ & 20 & Male & Bipolar disorder \\
\hline 18 & $H x$ & 41 & Male & $\begin{array}{c}\text { Schizophrenia } \\
\text { Drug abuse }\end{array}$ \\
\hline 19 & $1 x$ & 29 & Male & Bipolar disorder \\
\hline
\end{tabular}

